\title{
Czech dysplasia metatarsal type: another type II collagen disorder
}

\author{
Kristien P Hoornaert ${ }^{1}$, Ivo Marik ${ }^{2}$, Kazimierz Kozlowski ${ }^{3}$, Trevor Cole ${ }^{4}$, Martine Le Merrer $^{5}$, \\ Jules G Leroy ${ }^{1}$, Paul J Coucke ${ }^{1}$, David Sillence ${ }^{6}$ and Geert R Mortier ${ }^{*}, 1$ \\ ${ }^{1}$ Center for Medical Genetics, Ghent University Hospital, Ghent, Belgium; ${ }^{2}$ Ambulant Centre for Defects of Locomotor \\ Apparatus, Prague, Czech Republic; ${ }^{3}$ Department of Medical Imaging, The Children's Hospital at Westmead, Sydney, \\ Australia; ${ }^{4}$ Clinical Genetics Unit, Birmingham Women's Hospital, Birmingham, UK; ${ }^{5}$ Department of Medical \\ Genetics, Hopital Necker, Paris, France; ${ }^{6}$ Department of Medical Genetics, The Children's Hospital at Westmead, \\ Sydney, Australia
}

Czech dysplasia metatarsal type is an autosomal-dominant disorder characterized by an early-onset, progressive spondyloarthropathy with normal stature. Shortness of third and/or fourth toes is a frequently observed clinical feature. Similarities between individuals with this dysplasia and patients with an R275C mutation in the COL2A1 gene, prompted us to analyze the COL2A1 gene in the original families reported with Czech dysplasia. Targeted sequencing of exon 13 of the COL2A1 gene was performed, followed by sequencing of the remaining exons in case the R275C mutation was not identified. We identified the R275C substitution in two of the original patients reported with Czech dysplasia and three additional patients. All affected individuals had a similar phenotype characterized by normal height, spondyloarthropathy, short postaxial toes and absence of ocular and orofacial anomalies. The R275C mutation was excluded in a third patient reported with Czech dysplasia. However, the identification of the Y1391C mutation in this patient with disproportionate short stature made the diagnosis of

spondyloperipheral dysplasia (SPD) more probable. The Y1391C mutation is located in the C-propeptide of the procollagen chain and has been reported before in a patient with the Torrance type of lethal platyspondylic skeletal dysplasia (PLSD-T). Our observation of the same Y1391C mutation in an additional unrelated patient with SPD further supports the evidence that PLSD-T and SPD represent a phenotypic continuum. The R275C mutation in the COL2A1 gene causes a specific type II collagen disorder that was recently delineated as Czech dysplasia.

European Journal of Human Genetics (2007) 15, 1269-1275; doi:10.1038/sj.ejhg.5201913; published online 29 August 2007

Keywords: COL2A1; Czech dysplasia; spondyloperipheral dysplasia; arginine-to-cysteine mutations

\section{Introduction}

Czech dysplasia metatarsal type or progressive pseudorheumatoid arthritis with hypoplastic toes (OMIM no.

*Correspondence: Professor GR Mortier, Center for Medical Genetics, Ghent University Hospital, De Pintelaan 185, Ghent B-9000, Belgium.

Tel: + 32924036 03; Fax: + 32924049 70;

E-mail: geert.mortier@ugent.be

Received 1 March 2007; revised 18 May 2007; accepted 5 July 2007; published online 29 August 2007
609162) is an autosomal-dominant skeletal dysplasia first described in 2004 by Marik et al. ${ }^{1}$ Affected individuals have a normal stature but usually complain of severe joint pain before adolescence. Restricted mobility in the lower limb joints and a kyphoscoliosis are frequently observed. A typical phenotypic hallmark of the condition is shortening of the third and fourth toes, which is the result of metatarsal hypoplasia. Skeletal radiographs usually reveal signs of early osteoarthrosis in the spine and lower limb joints. Platyspondyly with irregular endplates and 
elongated vertebrae can be observed in the more severe cases. The upper extremities are usually normal; however, hand radiographs can reveal narrow joint spaces and flattened epiphyses. Up to now, five families from the Czech Republic have been reported with this disorder. ${ }^{1-3}$

Recently, we have shown that missense mutations in the COL2A1 gene, which substitute an arginine by a cysteine residue in the triple helical domain of the pro- $\alpha 1$ (II) collagen chain, cause a spectrum of disorders including Stickler syndrome, spondyloepiphyseal dysplasia congenita and spondyloarthropathy with brachydactyly. ${ }^{4}$ Affected individuals with the latter condition often have short third and fourth metatarsals and complain of arthropathy. The similarities between the latter phenotype and that in Czech dysplasia prompted the analysis of the COL2A1 gene and the specific search for arginine-to-cysteine mutations in the patients reported with Czech dysplasia and in three additional patients identified in the course of this study.

\section{Materials and methods Patients}

All patients were evaluated by at least one clinical geneticist at the referring center. The patients included in this study were the four original cases reported with Czech dysplasia $^{1,2}$ and three additional individuals that were identified during the course of this study with a phenotype resembling spondyloarthropathy with brachydactyly (as described in Hoornaert et $a l^{4}$ ) or Czech dysplasia. After informed consent, a blood or DNA sample was sent for mutation analysis of the COL2A1 gene.

\section{Molecular analysis}

Genomic DNA was extracted from blood samples by standard procedures, followed by touchdown PCR amplification. We started screening for the R275C substitution (c.823C $>\mathrm{T}$ mutation) in exon 13 of the COL2A1 gene since this particular nucleotide change is found in spondyloarthropathy with short metatarsals. ${ }^{4}$ (The R275C mutation corresponds to the $\mathrm{R} 75 \mathrm{C}$ mutation in the previous report by Hoornaert et al. ${ }^{4}$ The codon numbering has recently been changed with reading now starting at the first methionine (start codon for translation) instead of the first glycine residue in the triple helical domain of the proa1(II) collagen chain (GenBank accession no. L10347).) PCR amplification was performed using forward and reverse primers located in the flanking introns (primer sequences available upon request). The PCR products were analyzed by gel electrophoresis and visualized by ethidium bromide staining on $2 \%$ agarose gels. Sequences from our PCR products were obtained on the ABI PRISM 3730 automated sequencer (Applied Biosystems, Foster City, CA, USA) using the BigDye terminator cycle sequencing chemistry. The PCR products were compared to the wildtype sequence as submitted to GenBank accession no.
NM_001844. The nucleotides were numbered starting from the first base of the start codon (ATG) at position c. 158 of the cDNA reference sequence. Amino-acid residues were numbered from the start codon methionine (ATG) of the $\alpha 1$ (II) collagen chain (GenBank accession no. L10347).

If the $\mathrm{R} 275 \mathrm{C}$ mutation was not identified, the remaining 53 COL2A1 exons were analyzed by dHPLC using the WAVE DNA fragment analysis system (Transgenomic, Cheshire, UK). Oven temperatures were selected based on recommendations of the WAVEMAKER software program (Transgenomic). All fragments showing an aberrant pattern were directly sequenced as mentioned before.

\section{Results}

Targeted sequencing of exon 13 revealed that two of the four patients, previously reported with Czech dysplasia metatarsal type, ${ }^{1,2}$ were heterozygous for the c.823C $>\mathrm{T}$ mutation and hence carried the R275C substitution. This mutation was not found in 200 controls.

The first patient (RP0607547) in this study is case I in the paper by Marik et al. ${ }^{1}$ This 29 -year-old man with normal height but short trunk started complaining of joint pain and walking difficulties in early childhood. He had flexion contractures in the hip and knee joints and was confined to a wheelchair. Both feet showed short fourth and fifth toes. At the age of 27 years, he developed synovial osteochondromatosis of the knee. The radiographs showed flattened thoracic vertebral bodies with irregular endplates, deformed femoral heads and osteoarthritic changes in hands, hips and knees. The R275C mutation was also identified in his affected relatives.

The second patient (MV0607555) is the affected mother of case II in the paper by Kozlowski et al. ${ }^{2}$ She had chronic low back pain and had undergone bilateral hip replacement. She showed short third, fourth and fifth toes on both feet. Case II was not available for molecular testing but is clearly affected.

The R275C mutation was absent in case III (AK0607560) reported by Kozlowski et al. ${ }^{2}$ This girl also had short toes but differed from the other patients because of significant disproportionate short stature of prenatal onset. Her height at the age of 14 years was $134 \mathrm{~cm}(-4.5 S D)$. Molecular analysis revealed a heterozygous nucleotide change (c. $4172 \mathrm{~A}>\mathrm{G}$ ) in exon 53 of the C-propeptide, predicted to result in the substitution of tyrosine by cysteine at residue 1391 (p.Tyr1391Cys). In the last proband (FV0607551; case I in the paper by Kozlowski et al), no mutation was identified after sequencing of the entire COL2A1 gene. ${ }^{2}$ This individual did not have distinctive features as compared to the other cases with the R275C mutation.

In the course of this study, three additional unrelated patients were identified with a phenotype resembling 
Czech dysplasia. The first patient (AD0603929) is a 37-yearold woman of Serbian origin, who suffers since her teenage years from painful knees. She is of normal height $(167 \mathrm{~cm})$ and has short third and fourth metatarsals and short fourth metacarpals. Mild platyspondyly with irregular vertebral endplates, hip dysplasia and osteochondromatosis of the knees were demonstrated radiographically (Figure 1). The second patient (SB0304240), a woman of British origin, complains since early childhood of aching joints. At the age of 16 years, she was thought to have multiple epiphyseal dysplasia. She is of normal height but shows knee osteochondromatosis and short fourth metatarsals (Figure 2). The third patient (NH941702) is a 25-year-old Belgian woman suffering from walking difficulties and joint stiffness since childhood. She is of normal height $(171 \mathrm{~cm})$ and has short fourth toes. Radiographic evaluation revealed platyspondyly with irregular vertebral endplates, hip dysplasia and short fourth metatarsals (Figure 3). At the ages of 12 and 13 years, she had surgery in the right and left hip, respectively. In all three patients, the $\mathrm{R} 275 \mathrm{C}$ mutation was identified.

\section{Discussion}

In five unrelated patients with a similar phenotype, we have identified the same nucleotide change $(c .823 \mathrm{C}>\mathrm{T})$ in the COL2A1 gene. This mutation is predicted to result in the substitution of an arginine by cysteine residue (p.Arg275Cys) in the triple helical domain of the pro$\alpha 1$ (II) collagen chain. It does not involve a CpG dinucleotide and its occurrence is not due to a founder effect. Two of these affected individuals were the original patients reported with Czech dysplasia. The remaining three patients were identified and selected during the course of this study because of similar phenotypic features. These five patients have a normal stature and characteristic feet with short third, fourth and/or fifth toes. The major clinical morbidity is a severe arthropathy already starting in childhood and resulting in chronic pain in the back and the major joints of the lower limbs. The normal stature, normal intelligence and signs of arthropathy differentiate this condition from other disorders with postaxial toes such as pseudohypoparathyroidism (OMIM no. 103580) and the 2q37 deletion syndrome (OMIM no. 600430). ${ }^{5,6}$ Interestingly, cleft palate, myopia, vitreous anomalies and defective hearing, features often observed in type II collagen disorders, are absent in all five patients. Radiographic evaluation reveals mild platyspondyly with irregular vertebral endplates, narrowed intervertebral spaces and sometimes elongated vertebrae. Signs of osteoarthrosis are mainly visible in the lower limbs with deformed femoral heads and dysplastic pelvic changes. The metatarsal hypoplasia involving the postaxial toes is a diagnostically important and distinctive feature. Five additional
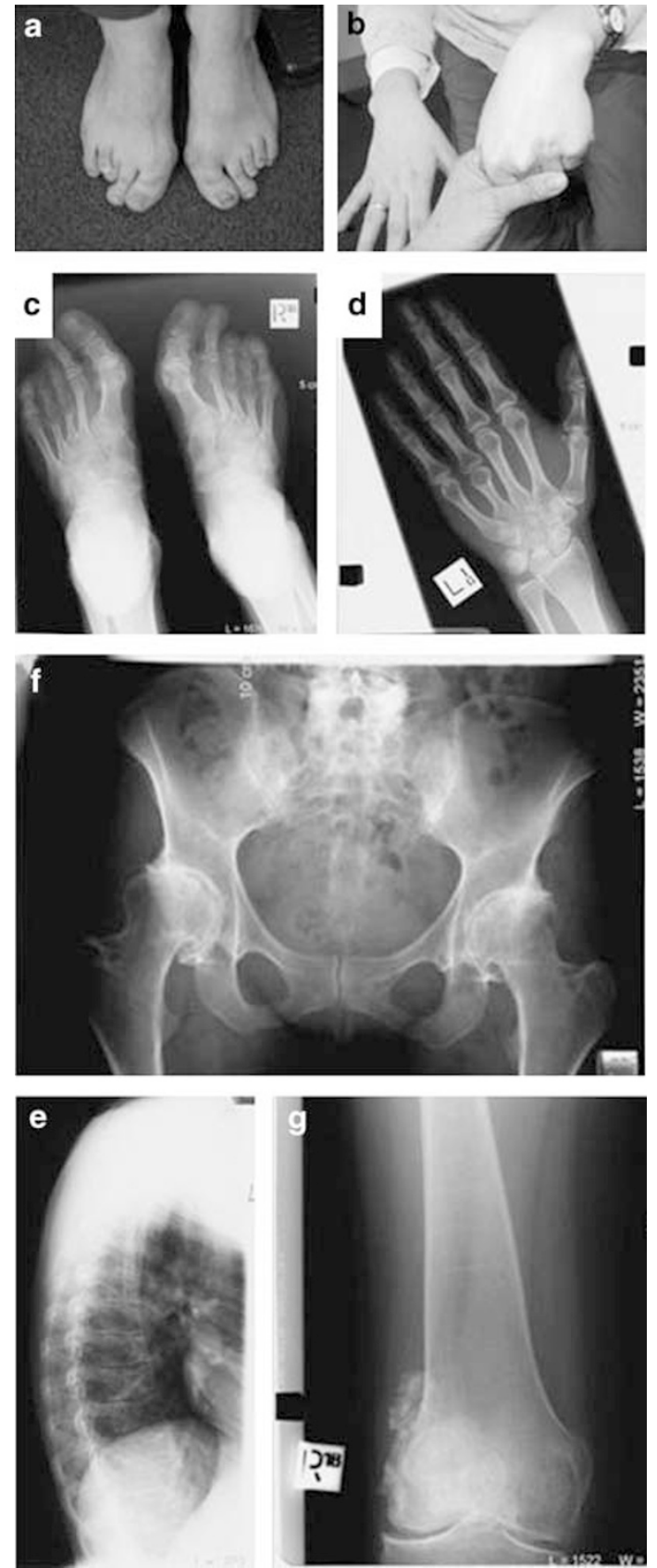

Figure 1 Clinical and radiographic pictures of patient AD0603929. There is shortening of the third to fifth toes on both feet (a). Brachydactyly with absent knuckle sign for the fourth finger is shown on the left hand (b). Anteroposterior radiographs of both feet reveal shortening of mainly the fourth and fifth metatarsals (c). The short fourth metacarpal is visible on the left-hand radiograph (d). Platyspondyly with irregular and sunken endplates is seen on the lateral radiograph of the thoracic spine (e). Anteroposterior radiographs of pelvis and right knee show respectively coxarthrosis (f) and osteochondromatosis lesions in the knee joint (g).

patients with this $\mathrm{R} 275 \mathrm{C}$ mutation have been reported in the literature and all of them share the same clinical and radiographic features (Table 1$)^{4,7-11}$ 


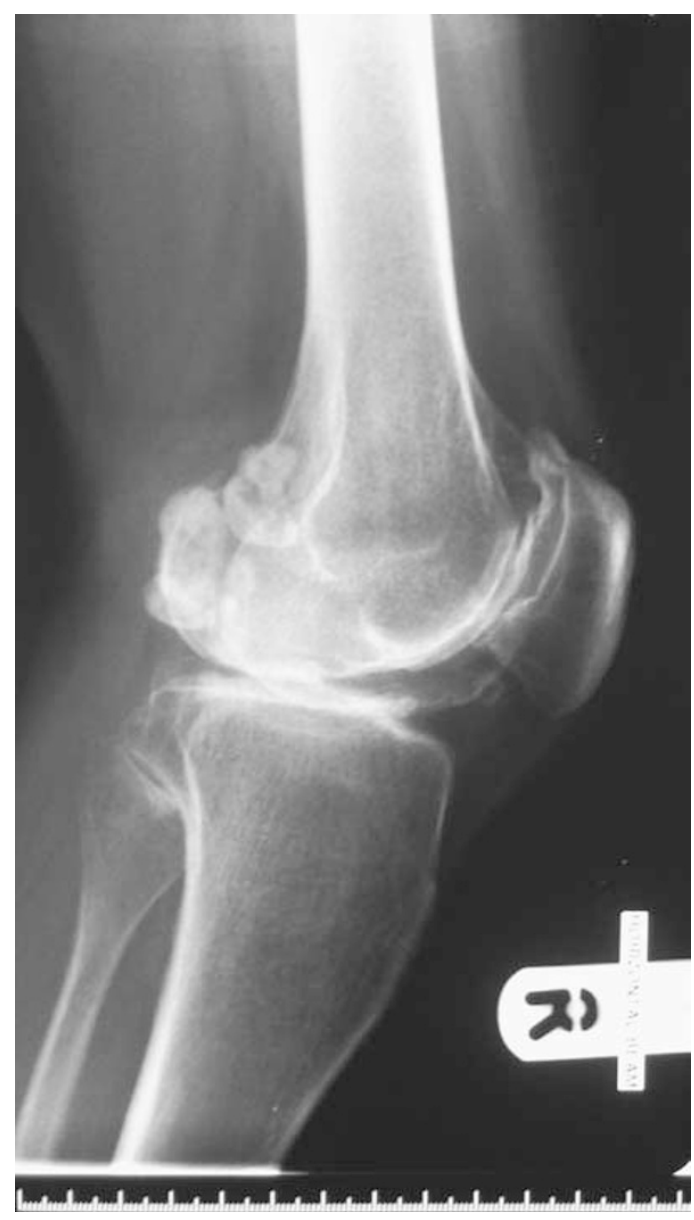

Figure 2 Lateral radiograph of the right knee in patient SB0304240 shows osteochondromatosis lesions in the posterior part of the joint.

There is evidence that substituting a hydrophilic arginine by a hydrophobic cysteine within the triple helical domain of the collagen protein affects the integrity and stability of the collagen fibril. Previous analyses have shown that arginine is the most stabilizing residue in the Y-position of the Gly-X-Y repeat. ${ }^{12}$ On the basis of the collagen stability algorithm (http://jupiter.umdnj.edu/ collagen_calculator/), we can calculate that the R275C mutation causes local destability of the procollagen chains $\left(\Delta T=1.22^{\circ} \mathrm{C}\right) .{ }^{13}$ However, Steplewski et al ${ }^{14}$ did not notice any change in thermostability of the entire pro- $\alpha 1$ (II) collagen chain containing R275C. This observation suggests that the mutation only affects local thermostability without adverse effect upon the entire collagen chain. Cysteine residues are absent in the triple helical domain of the pro- $\alpha 1$ (II) collagen chain. ${ }^{12,15}$ When introduced by mutation, cysteine residues may engage in creating either intramolecular or intermolecular disulfide bonds and hence unfavorably affect the supramolecular conformation of the collagen fibrillar network. ${ }^{16}$ Cartilage analysis in a patient with the $\mathrm{R} 275 \mathrm{C}$ mutation has shown that the fibrillar network is abnormally organized and composed of thin appearing fibrils. ${ }^{8}$

In one patient (AK0607560), reported previously to be affected by Czech dysplasia, the R275C mutation was excluded. This patient was however heterozygous for a c. $4172 \mathrm{~A}>\mathrm{G}$ transition resulting in the substitution of tyrosine by cysteine (Y1391C) in the C-propeptide of the pro- $\alpha 1$ (II) collagen. Disproportionate short stature was the most distinct feature between this individual and the ones with the R275C mutation. We believe that this individual has spondyloperipheral dysplasia (SPD), another type II collagen disorder that resembles spondyloepiphyseal dysplasia congenita both clinically and radiographically but differs from it by the brachydactyly. ${ }^{17-21}$ The Y1391C mutation has been published before in a patient with lethal platyspondylic skeletal dysplasia, Torrance type (PLSD-T). ${ }^{22}$ In rare instances of survival, infants with PLSD-T develop a phenotype resembling SPD, suggesting that both conditions are not separate disorders but rather represent a phenotypic continuum..$^{23-25}$ Our recent identification of the same Y1391C mutation in another unrelated patient with SPD (data not shown) underscores the causality of this mutation and supports the hypothesis that both SPD and PLSD-T share a common pathogenetic mechanism. Both conditions are caused exclusively by mutations in the C-propeptide of the pro- $\alpha 1$ (II) collagen chain. In PLSD-T both truncating and missense mutations have been found, ${ }^{23-25}$ whereas in SPD only truncating mutations have been reported..$^{20,21,23,26}$ The two patients in this study, however, show that SPD can also result from missense mutations in the C-propeptide (Figure 4). The C-propeptide of the pro- $\alpha 1$ (II) collagen chain contains eight cysteine residues. The integrity of the C-propeptide is critical for proper chain association and for subsequent folding of the individual chains into a collagen trimer with triple helical configuration. The cysteine residues play an important role in this process through formation of intra- and interchain disulfide bonds. ${ }^{27}$ Intrachain disulfide bonds are formed between cysteine residues 1393 and 1438 and the cysteine residues 1323 and 1485, respectively. Cysteine residues 1283, 1289, 1306 and 1315 are involved in interchain disulfide bonding. Truncated chains lacking a substantial part of the C-propeptide are hardly or not incorporated into the collagen trimer. ${ }^{21,27}$ Mutations that introduce a new cysteine residue within the C-propeptide may have a similar deleterious effect on chain association and trimer formation. By introducing a cysteine residue in exon 53, close to C1393, the Y1391C mutation may disturb normal intrachain disulfide bond formation.

Brachydactyly and disproportionate short stature are important phenotypic consequences of mutations in the C-propeptide of the pro- $\alpha 1$ (II) collagen chain. However, not all mutations in this domain will result in a short 

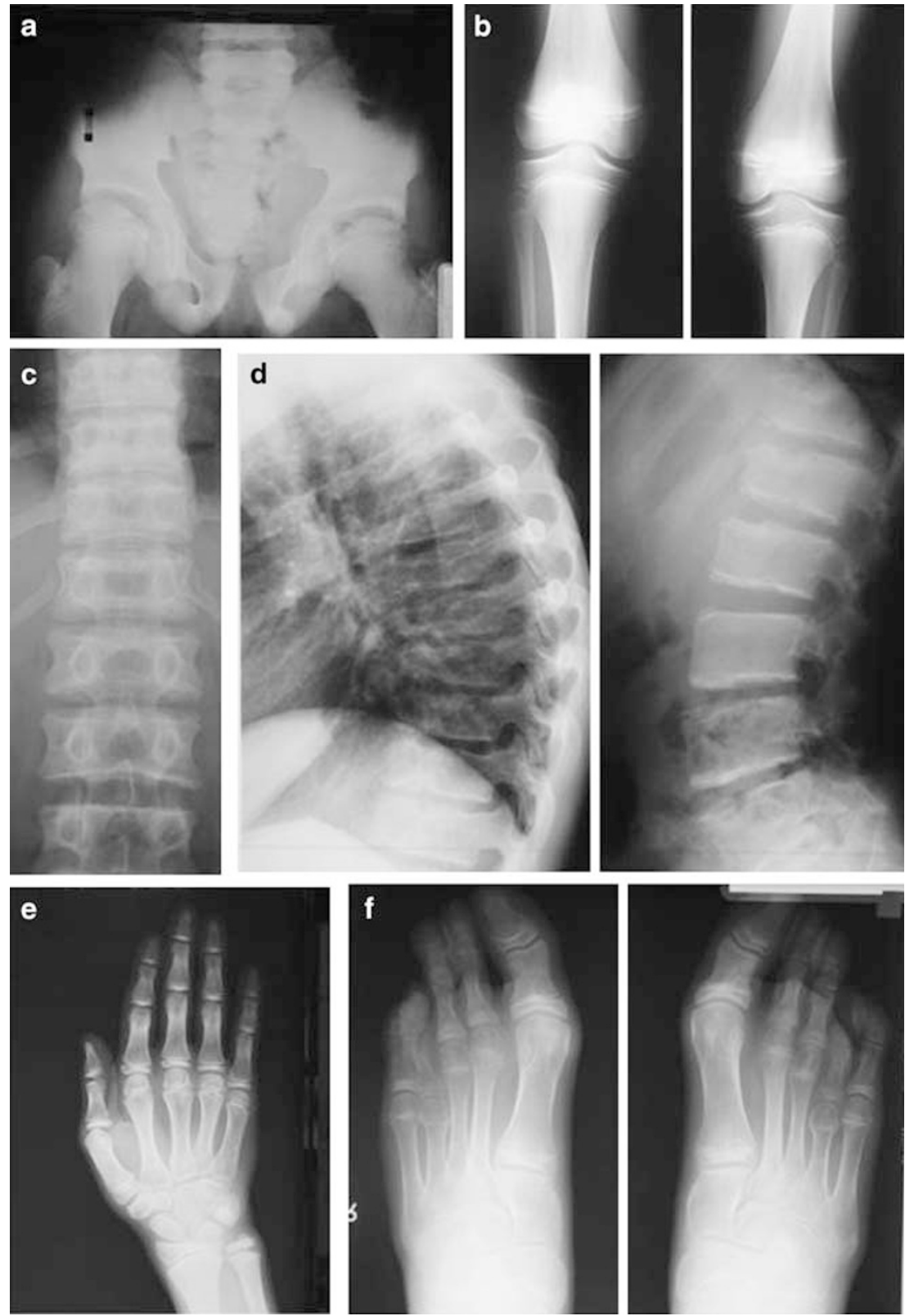

Figure 3 Radiographs of patient NH941702 taken at different ages. Anteroposterior radiograph of the pelvis at the age of 7 years 7 months reveals small and broad iliac wings, irregular acetabular contours and small proximal femoral epiphyses (a). The knees at the age of 7 years 10 months are mildly affected with convexity of the proximal tibial growth plate (b). Radiographs of the spine at the age of 12 years 7 months show medially placed pedicles ('open staircase vertebral bodies') on the anteroposterior pictures (c) and platyspondyly with irregular endplates on the profile films. Mainly the thoracic vertebrae have an elongated appearance (d). The right-hand film at the age of 12 years 7 months shows shortening of all metacarpals and phalanges with rather broad diaphyses, helmet-shaped metacarpal epiphyses and large carpal bones (e). The feet at the same age show bilateral shortening mainly the fourth metatarsal and broad metatarsal and phalanges in the great toe (f).

stature phenotype. A previously published patient with the R1276C mutation had brachydactyly and spondyloarthropathy but with normal height. ${ }^{4}$ Furthermore, the G1305D mutation, which has been reported in a patient with vitreoretinopathy and phalangeal epiphyseal dysplasia, was also associated with a normal stature. ${ }^{28}$ In comparison to the other C-propeptide mutations causing short stature, these two mutations are located more proximal in the C-propeptide of the pro- $\alpha 1$ (II) collagen chain. Hence, it seems that mutations located around the cysteine residues involved in intrachain disulfide bonding have a more deleterious effect on growth than the ones located more 
Table 1 Clinical and radiographic features in patients with the R275C mutation in the COL2A1 gene

\begin{tabular}{|c|c|c|c|c|c|c|}
\hline & \multicolumn{5}{|c|}{ Our series $(\mathrm{n}=5)$} & \multirow[b]{2}{*}{$\begin{array}{l}\text { Literature }^{4,7-11} \\
\quad(\mathrm{n}=5)\end{array}$} \\
\hline & $\begin{array}{l}\text { RP0607547 (I) } \\
\text { Czech Republic }\end{array}$ & $\begin{array}{l}\text { MV0607555 (II) } \\
\text { Czech Republic }\end{array}$ & $\begin{array}{l}\text { AD0603929 } \\
\text { Serbia }\end{array}$ & $\begin{array}{l}\text { SB0304240 } \\
\text { United Kingdom }\end{array}$ & $\begin{array}{l}\text { NH941702 } \\
\text { Belgium }\end{array}$ & \\
\hline \multicolumn{7}{|l|}{ Clinical features } \\
\hline \multicolumn{7}{|l|}{ Origin } \\
\hline Normal height & + & + & + & + & + & $5 / 5$ \\
\hline $\begin{array}{l}\text { Ocular anomalies } \\
\text { (myopia, retinal detachment) }\end{array}$ & - & - & - & - & - & $0 / 5$ \\
\hline Hearing loss & - & - & - & - & - & $2 / 5$ \\
\hline Cleft palate & - & - & - & - & - & $0 / 5$ \\
\hline Joint pain in childhood & + & + & + & + & + & $5 / 5$ \\
\hline Limited joint mobility & + & + & + & + & + & $5 / 5$ \\
\hline Short toes (III-IV) & + & + & + & + & + & $5 / 5$ \\
\hline $\begin{array}{l}\text { Hip replacement before } \\
\text { the age of } 36 \text { years }\end{array}$ & - & + & - & + & + & $4 / 5$ \\
\hline \multicolumn{7}{|l|}{ Radiographic features } \\
\hline \multicolumn{7}{|l|}{ Spine } \\
\hline Platyspondyly & + & + & + & + & + & $5 / 5$ \\
\hline Irregular vertebral plates & + & + & + & + & + & $5 / 5$ \\
\hline \multicolumn{7}{|l|}{ Hands/feet } \\
\hline Short metatarsals (III-IV) & + & + & + & + & + & $5 / 5$ \\
\hline Short metacarpals (IV-V) & - & - & + & - & + & $1 / 5$ \\
\hline \multicolumn{7}{|l|}{ Limbs } \\
\hline Osteoarthrosis & + & + & + & + & + & $5 / 5$ \\
\hline Osteochondromatosis & + & - & + & + & - & $4 / 5$ \\
\hline
\end{tabular}

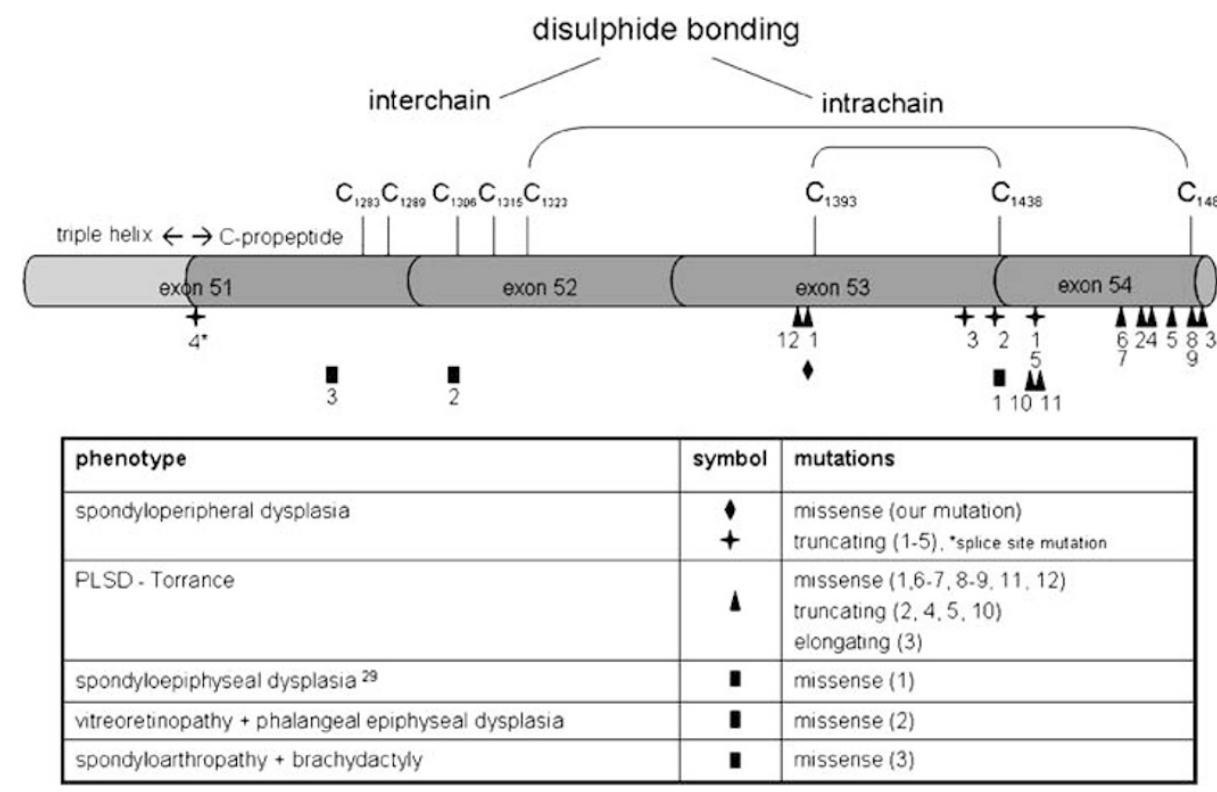

Figure 4 Schematic representation of the C-propeptide of the pro- $\alpha 1$ (II) collagen chain with indication of the cysteine residues involved in intraand interchain disulfide bonding and localization of mutations with the corresponding phenotypes.

proximal, in the neighbourhood of cysteine residues necessary for the formation of interchain disulfide bonds.

In conclusion, we have shown that the R275C mutation in the COL2A1 gene is a recurrent mutation causing a specific phenotype within the type II collagen disorders. The disorder is characterized by a spondyloarthropathy with normal stature and lack of orofacial anomalies. Shortening of postaxial toes is a remarkable feature that 
should prompt the clinician to consider this diagnosis in patients with chronic and early-onset osteoarthrosis. In addition, we have shown that patients, previously reported with Czech dysplasia, have this mutation. We therefore suggest that Czech dysplasia should be incorporated as a new member within the family of type II collagenopathies. Both Czech dysplasia and 'spondyloarthropathy with short third and fourth toes' should now be considered as one and the same disorder. We also have provided further evidence that both SPD and PLSD-T represent a phenotypic continuum since the same missense mutation in the $\mathrm{C}$ propeptide may cause either disorder. Finally, we have observed a relationship between the localization of missense mutations in the C-propeptide and the resulting phenotype when linear growth and final stature are taken into account.

\section{Acknowledgements}

We are indebted to the families and patients for their interest and cooperation. This study is supported, in part, by the Fund for Scientific Research, Flanders, with a mandate fundamental clinical research and a research grant (no. G.0331.03) to GRM and by the Fifth Framework of the specific research and technological development program 'Quality of Life and Management of Living Resources' of the European Commission (Contract QLG1-CT-2001-02188).

\section{References}

1 Marik I, Marikova O, Zemkova D, Kuklik M, Kozlowski K: Dominantly inherited progressive pseudorheumatoid dysplasia with hypoplastic toes. Skeletal Radiol 2004; 33: 157-164.

2 Kozlowski K, Marik I, Marikova O, Zemkova D, Kuklik M: Czech dysplasia metatarsal type. Am J Med Genet 2004; 129: 87-91.

3 Marik I, Marikova O, Zemkova D, Kuklik M, Kozlowski K: Czech dysplasia metatarsal type. Hungarian Radiol 2005; 79: 89-93.

4 Hoornaert KP, Dewinter C, Vereecke I et al: The phenotypic spectrum in patients with arginine to cysteine mutations in the COL2A1 gene. J Med Genet 2006; 43: 406-413.

5 Fitch N: Albright's hereditary osteodystrophy: a review. Am J Med Genet 1982; 11: 11-29.

6 Wilson LC, Leverton K, Oude Luttikhuis ME et al: Brachydactyly and mental retardation: an Albright hereditary osteodystrophy-like syndrome localized to 2q37. Am J Hum Genet 1995; 56: 400-407.

7 Bleasel JF, Holderbaum D, Mallock V, Haqqi TM, Williams HJ, Moskowitz RW: Hereditary osteoarthritis with mild spondyloepiphyseal dysplasia - are there 'hot spots' on COL2A1? J Rheumatol 1996; 23: 1594-1598.

8 Bleasel JF, Bisagni-Faure A, Holderbaum D et al: Type II procollagen gene (COL2A1) mutation in exon 11 associated with spondyloepiphyseal dysplasia, tall stature and precocious osteoarthritis. J Rheumatol 1995; 22: 255-261.

9 Lopponen T, Korkko J, Lundan T, Seppanen U, Ignatius J, Kaariainen H: Childhood-onset osteoarthritis, tall stature, and sensorineural hearing loss associated with Arg75-Cys mutation in procollagen type II gene (COL2A1). Arthritis Rheum 2004; 51: 925-932.

10 Reginato AJ, Passano GM, Neumann G et al: Familial spondyloepiphyseal dysplasia tarda, brachydactyly, and precocious osteoar- thritis associated with an arginine $75 \rightarrow$ cysteine mutation in the procollagen type II gene in a kindred of Chiloe Islanders. I. Clinical, radiographic, and pathologic findings. Arthritis Rheum 1994; 37: 1078-1086.

11 Williams CJ, Considine EL, Knowlton RG et al: Spondyloepiphyseal dysplasia and precocious osteoarthritis in a family with an Arg $75 \rightarrow$ Cys mutation in the procollagen type II gene (COL2A1). Hum Genet 1993; 92: 499-505.

12 Persikov AV, Ramshaw JA, Brodsky B: Collagen model peptides: sequence dependence of triple-helix stability. Biopolymers 2000; 55: 436-450.

13 Persikov AV, Ramshaw JA, Brodsky B: Prediction of collagen stability from amino acid sequence. I Biol Chem 2005; 280: $19343-19349$.

14 Steplewski A, Majsterek I, McAdams E et al: Thermostability gradient in the collagen triple helix reveals its multi-domain structure. J Mol Biol 2004; 338: 989-998.

15 Kielty C, Grant M: The collagen family: structure, assembly, and organization in the extracellular matrix; in Royce PM, Steinmann B (eds): Connective Tissue and Its Heritable Disorders 2002, Wiley-Liss: New York, pp 159-222.

16 Steplewski A, Ito H, Rucker E et al: Position of single amino acid substitutions in the collagen triple helix determines their effect on structure of collagen fibrils. J Struct Biol 2004; 148: 326-337.

17 Sorge G, Ruggieri M, Lachman RS: Spondyloperipheral dysplasia. Am J Med Genet 1995; 59: 139-142.

18 Kelly TE, Lichtenstein JR, Dorst JP: An unusual familial spondyloepiphyseal dysplasia: 'spondyloperipheral dysplasia'. Birth Defects Orig Artic Ser 1977; 13: 149-165.

19 Vanek J: Spondyloepiphyseal dysplasia. J Med Genet 1983; 20: 117-121.

20 Zabel B, Hilbert K, Stoss H, Superti-Furga A, Spranger J, Winterpacht A: A specific collagen type II gene (COL2A1) mutation presenting as spondyloperipheral dysplasia. Am J Med Genet 1996; 63: 123-128.

21 Zankl A, Zabel B, Hilbert K et al: Spondyloperipheral dysplasia is caused by truncating mutations in the C-propeptide of COL2A1. Am J Med Genet 2004; 129: 144-148.

22 Nishimura G, Nakashima E, Mabuchi A et al: Identification of COL2A1 mutations in platyspondylic skeletal dysplasia, Torrance type. I Med Genet 2004; 41: 75-79.

23 Nishimura G, Haga N, Kitoh $\mathrm{H}$ et al: The phenotypic spectrum of COL2A1 mutations. Hum Mut 2005; 26: 36-43.

24 Nishimura G, Nakashima E, Mabuchi A et al: Identification of COL2A1 mutations in platyspondylic skeletal dysplasia, Torrance type. J Med Genet 2004; 41: 75-79.

25 Zankl A, Neumann L, Ignatius $J$ et al: Dominant negative mutations in the C-propeptide of COL2A1 cause platyspondylic lethal skeletal dysplasia, Torrance type, and define a novel subfamily within the type 2 collagenopathies. Am J Med Genet 2005; 133: 61-67.

26 Schell T, Kulozik AE, Hentze MW: Integration of splicing, transport and translation to achieve mRNA quality control by the nonsense-mediated decay pathway. Genome Biol 2002; 3: Reviews 1006.1-1006.6.

27 Hulmes DJ: Building collagen molecules, fibrils, and suprafibrillar structures. J Struct Biol 2002; 137: 2-10.

28 Richards AJ, Morgan J, Bearcroft PW et al: Vitreoretinopathy with phalangeal epiphyseal dysplasia, a type II collagenopathy resulting from a novel mutation in the C-propeptide region of the molecule. J Med Genet 2002; 39: 661-665.

29 Unger S, Korkko J, Krakow D, Lachman RS, Rimoin DL, Cohn DH: Double heterozygosity for pseudoachondroplasia and spondyloepiphyseal dysplasia congenita. Am J Med Genet 2001; 104: 140-146. 